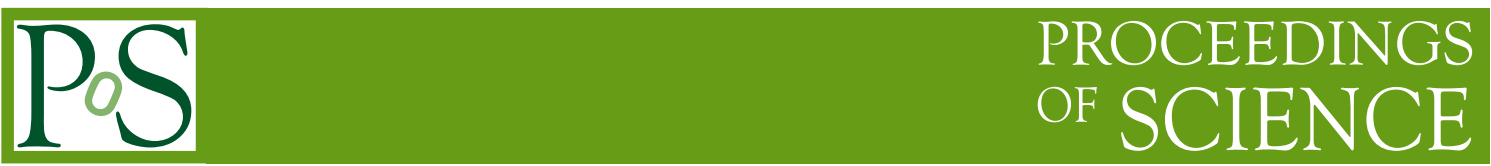

\title{
ATLAS Higgs Searches
}

\section{Henso Abreu* on behalf of the ATLAS Collaboration}

Laboratoire de l'Accélérateur Linéaire, IN2P3/CNRS, Université Paris-Sud 11, Orsay, France.

E-mail: abreu@lal.in2p3.fr

A search for the Standard Model Higgs boson at the Large Hadron Collider (LHC) running at a centre-of-mass energy of $7 \mathrm{TeV}$ is reported, based on a total integrated luminosity of 1.0 to $2.3 \mathrm{fb}^{-1}$ collected by the ATLAS detector in 2011. A number of Higgs boson decay channels: $W H \rightarrow l v b \bar{b}, Z H \rightarrow l l b \bar{b}, H \rightarrow \tau^{+} \tau^{-}, H \rightarrow \gamma \gamma, H \rightarrow W W^{(*)} \rightarrow l v l v, H \rightarrow W W \rightarrow l v q q, H \rightarrow$ $Z Z^{(*)} \rightarrow l l l l, H \rightarrow Z Z \rightarrow l l v v$ and $H \rightarrow Z Z \rightarrow l l q q(l$ is $e, \mu$ and $q$ can be any of $u, d, s, c$ or $b)$ are combined in a mass range from $110 \mathrm{GeV}$ to $600 \mathrm{GeV}$. No significant evidence for a signal is observed. The Standard Model Higgs boson mass ranges from $146 \mathrm{GeV}$ to $232 \mathrm{GeV}, 256 \mathrm{GeV}$ to $282 \mathrm{GeV}$ and $296 \mathrm{GeV}$ to $466 \mathrm{GeV}$ are excluded at the $95 \% \mathrm{CL}$, while the expected Standard Model Higgs boson exclusion in the absence of signal ranges between $131 \mathrm{GeV}$ to $447 \mathrm{GeV}$. In addition, the search for the neutral Higgs bosons in the Minimal Supersymmetric extension to the Standard Model (MSSM) is presented, where exclusion limits at the 95\% CL are derived for the production cross section of a generic Higgs boson $\phi$ as a function of the Higgs boson mass and for $A / H / h \rightarrow \tau^{+} \tau^{-}$in the MSSM as a function of the parameters $m_{A}$ and $\tan \beta$ in the $m_{h}^{\max }$ scenario.

The XXth International Workshop High Energy Physics and Quantum Field Theory September 24-October 1, 2011

Sochi Russia

${ }^{*}$ Speaker. 


\section{Introduction}

The search for the Higgs boson, both in the Standard Model (SM) and Beyond SM scenarios is one of the keys aims of the Large Hadron Collider (LHC) at CERN. Although the Higgs boson is not discovered yet, limits have been set on its mass by LEP [1] and Tevatron [2,3] experiments, excluding, in the case of the SM scenario, a Higgs boson below $114.4 \mathrm{GeV}$ and in the range of $158-175 \mathrm{GeV}$ at $95 \%$, while current indirect constraints from electroweak fit provides a most probable mass of $120_{-5}^{+12} \mathrm{GeV}$ [4]. The results from the ATLAS experiment are available in various SM Higgs boson search channels [5-12], which are outlined in Section 4. The combined results are shown in Section 5, it is based on the profile likelihood ratio technique [13] as a test statistic. In section 6 , the search for neutral MSSM Higgs bosons in the decay mode $A / H / h \rightarrow \tau^{+} \tau^{-}$is presented and the conclusions are drawn in Section 7.

\section{Standard Model Higgs boson at the LHC}
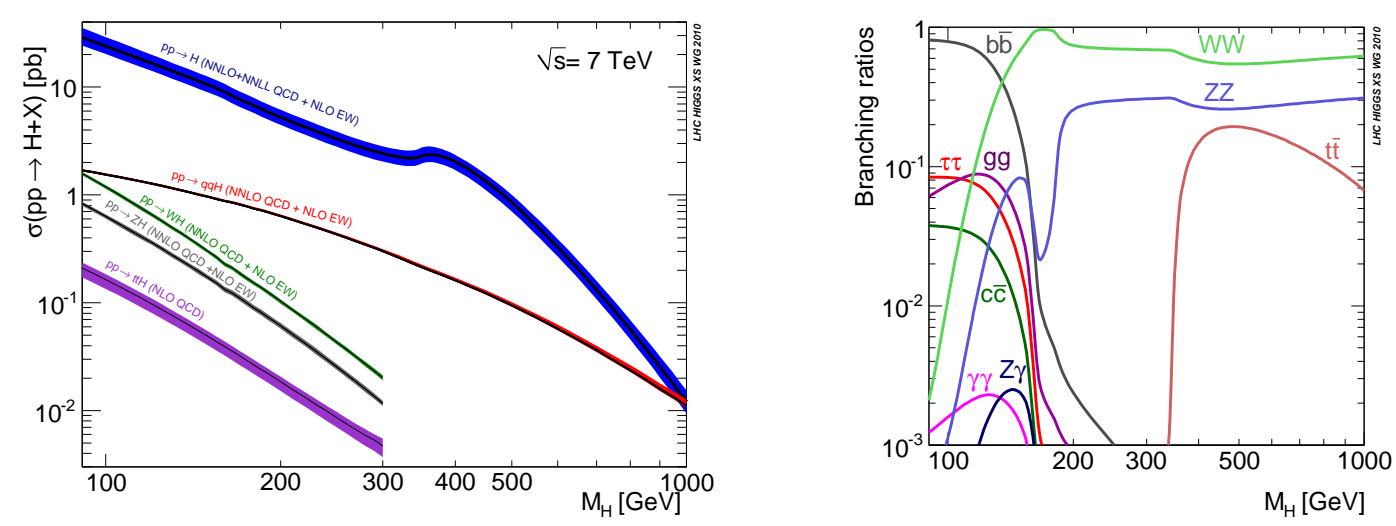

Figure 1: Left: SM Higgs boson production cross section and (right) branching ratios as a function of $M_{H}$ at the LHC at $\sqrt{s}=7 \mathrm{TeV}[14]$.

\subsection{The Higgs production}

In proton-proton collisions at the LHC the SM Higgs boson will be produced by the following processes, (for details see [14]) ordered by cross section (see Figure 1):

Gluon fusion. The Higgs boson is produced by the fusion of two gluons via a heavy-quark loop ${ }^{1}$. This process is also called direct production and it has the largest cross section over the allowed mass range. Important higher order processes are for example $g g \rightarrow H, g q \rightarrow H q, g \bar{q} \rightarrow$ $H g$. The NNLO cross section was computed in the last 10 years [15-17] and there is currently a large debate on the present state of understanding of the theoretical uncertainties (currently thought to be around $15 \%$ ).

Vector-Boson-Fusion $(V B F)$. In this channel the Higgs boson is produced by the fusion of two weak vector bosons ( $W$ or $Z$ ) which are radiated off quarks. This process leaves a special signature

\footnotetext{
${ }^{1}$ The loop is dominated by top quarks.
} 
in the detector. The quarks hadronize to jets which will be detected in the forward region of the detector (close to the beam pipe). There is no color connection between the two quarks, hence between the two forward jets, little hadronic activity is expected in the signal process. This typical VBF signature is used to suppress the QCD background. This process has been computed recently at NNLO [18].

Associated production $W^{ \pm} H, Z H$. In this process the Higgs boson is radiated off a weak boson (Higgstrahlung). Its cross section falls rapidly an increasing value of $M_{H}$ and has been computed at NNLO [19].

Associated production $t \bar{t} H$. The Higgs production in association with top-pairs is less important because the cross section is about five times smaller than the one for $W^{ \pm} H$ or $Z H$ for the low mass range $M_{H}$. It has been computed at NLO [20-22].

\subsection{The Higgs decay modes}

In this subsection, the three main mass regions (see Figure 1) to be exploited at the LHC will be described.

In the low mass range $\left(100 \leq M_{H} \lesssim 130 \mathrm{GeV}\right)$ the Higgs boson mainly decays into a $b \bar{b}$ pair, $B R_{b \bar{b}}>70-75 \%$, due to the Higgs coupling to fermions proportional to their mass. The discovery potential of Higgs search in this channel at LHC is however small due to large QCD background. The $H \rightarrow \tau^{+} \tau^{-}$channel has a smaller branching ratio, about $8 \%$, and suffers from large background from the $Z \rightarrow \tau^{+} \tau^{-}$decay process. However, when searching for the Higgs in the VBF production mode, this is a promising channel at low masses. The $\gamma \gamma$ and $Z \gamma$ decay modes also occur in the low mass range and are due to the indirect Higgs coupling to the photon. Despite its small branching ratio, the $H \rightarrow \gamma \gamma$ is a very important channel at low masses, $\left(100<M_{H}<150 \mathrm{GeV}\right)$ because it presents a very clean signature and has a narrow width which will provide sufficient discrimination against backgrounds.

In the intermediate mass range $\left(130 \leq M_{H} \lesssim 200 \mathrm{GeV}\right)$. The $W W^{(*)}$ and $Z Z^{(*)}$ channels are open and have a large branching ratio due to their stronger coupling to the Higgs. The decay to $W W^{(*)}$ is the most promising channel, because this decay has a branching ratio close to $100 \%$ in this mass range. The $Z Z^{(*)} \rightarrow 4 l$ is a very important channel at medium to high masses $\left(M_{H} \geq 200\right.$ $\mathrm{GeV}$ ), since the presence of four electrons or muons in one event is a very unique and therefore distinguishable signature at a hadron collider.

At $M_{H}>2 M_{t o p} \sim 350 \mathrm{GeV}$, the top pair decay opens and increases rapidly due its very strong coupling to the Higgs. However this channel suffers from large QCD and $t \bar{t}$ backgrounds. There may be however some possibilities in the channels $H \rightarrow Z Z \rightarrow l l v v$ and $H \rightarrow Z Z \rightarrow l l q q$ and $H \rightarrow W W \rightarrow l v q q$.

\section{A Toroidal LHC ApparatuS}

The ATLAS detector [23] consists of several subsystems. An inner tracking detector is immersed in a 2 Tesla magnetic field produced by a superconducting solenoid. Charged particle position measurements are made by silicon detectors in the pseudorapidity range $|\eta|<2.5$ and 
by a straw tube tracker in the range $|\eta|<2.0^{2}$. The calorimeters cover $|\eta|<4.9$ with a variety of detector technologies. The lead liquid-argon electromagnetic (EM) calorimeter is divided into barrel $(|\eta|<1.475)$ and endcap $(1.375<|\eta|<3.2)$ regions. The EM calorimeter consists of three longitudinal layers with the first (strip) having the highest granularity in the $\eta$ direction, and the second collecting most of the electromagnetic shower energy. A thin presampler layer covering the range $|\eta|<1.8$ is used to correct for the energy lost by EM particles upstream of the calorimeter. The hadronic calorimeters (using liquid argon or scintillating tiles as active materials) surround the EM calorimeter and cover $|\eta|<4$.9. The muon spectrometer measures the deflection of muon tracks in the field of three large superconducting toroid magnets. It is instrumented with separate trigger $(|\eta|<2.4)$ and high-precision tracking $(|\eta|<2.7)$ chambers.

\section{Standard Model Higgs boson searches}

\section{1 $H \rightarrow b \bar{b}:$ The $W / H(l v b \bar{b})$ and $Z / H(l l b \bar{b})$}

At the LHC the Higgs boson will decay into two $b$-quarks with a high branching ratio (Figure 1), but the background to $H \rightarrow b \bar{b}$ from SM processes is expected to be prohibitively large. However, the production of Higgs boson in association with an electroweak boson $V H$ (where $V=Z$ or $W$ ) is likely to be a more promising process to identify $H \rightarrow b \bar{b}$ decays due to the reduction in background. So, the analysis of the direct search for the SM Higgs boson when produced in association with a $W$ or $Z$ boson decaying to $b \bar{b}$ is performed using a dataset corresponding to an integrated luminosity of $1.04 \mathrm{fb}^{-1}[5]$.

The $Z H \rightarrow l l b \bar{b}$ and $W H \rightarrow l v b \bar{b}$ analyses both use single lepton triggers with a $p_{T}$ threshold of $18 \mathrm{GeV}$ for muons and $20 \mathrm{GeV}$ for electrons. In the $Z H \rightarrow l l b \bar{b}$ search channel the selection continues with the requirement of a $Z$ candidate and small $E_{T}^{\text {miss }}$. The leptonic $Z$ decay candidates are selected by requiring the invariant mass of the two leptons to lie within the range $76<m_{l l}<$ $106 \mathrm{GeV}$ (approximately $\pm 15 \mathrm{GeV}$ around the mass of the $Z$ boson). This strongly suppresses background from events without a real $Z$ boson, such as $t \bar{t}$ and multijet production. Moreover, at least two jets with $p_{T}>25 \mathrm{GeV}$ are required where the two highest $p_{T}$ jets are both required to pass the $b$-tagging selection. In the $l l b \bar{b}$ channel the dominant background is expected to be from $Z+$ jets events. Its normalization is estimated in a control region sample in the sidebands defined by $m_{b \bar{b}}<80 \mathrm{GeV}$ and $140<m_{b \bar{b}}<250 \mathrm{GeV}$ and its shape is taken from the Monte Carlo simulation. Other sources of background, among which the most relevant are top and diboson production, are estimated by Monte Carlo simulation. The background coming from top was also checked in the sidebands of the dilepton invariant mass distribution.

For the $W H \rightarrow l v b \bar{b}$ events are characterized by a lepton originating from $W$ decay and significant $E_{T}^{\text {miss }}$. So, events are required to have exactly one lepton ( $e$ or $\mu$ ). The missing transverse energy $E_{T}^{\text {miss }}$ is required to be greater than $25 \mathrm{GeV}$ to reduce background from events without large real $E_{T}^{\text {miss }}$ such as $Z$ decay to leptons, when the leptons escapes detection, and QCD multijet background. In addition, the number of jets with $p_{T}>25 \mathrm{GeV}$ is required to be exactly two to reduce

\footnotetext{
${ }^{2}$ ATLAS uses a right-handed coordinate system with its origin at the nominal interaction point (IP) in the centre of the detector and the $z$-axis coinciding with the axis of the beam pipe. The $x$-axis points from the IP to the centre of the LHC ring, and the $y$-axis points upward. Cylindrical coordinates $(r, \phi)$ are used in the transverse plane, $\phi$ being the azimuthal angle around the beam pipe. The pseudorapidity is defined in terms of the polar $\theta$ as $\eta=-\ln \left(\tan \frac{\theta}{2}\right)$.
} 
background from top-quark production that is significantly higher in this channel. The two jets must pass the $b$-tagging selection. The remaining contribution from top and $W+$ jets background is determined by a simultaneous template fit to the $m_{b \bar{b}}$ distribution in the signal-free control regions of $m_{b \bar{b}}<80 \mathrm{GeV}$, where $W+$ jets dominates, and $140<m_{b \bar{b}}<250 \mathrm{GeV}$ which is dominated by the top background. The small contribution from other background is subtracted from the $m_{b \bar{b}}$ distribution before the fit. The top and $W+$ jets templates are determined from Monte Carlo simulation for $t \bar{t}$ events and un-tagged $W+$ jets events from data. Relaxing the two-jet requirement to three jets provides a control sample dominated by top-pair production which is used to check its shape and normalization. Both were found to be well described. Finally, the multi-jet background, which contributes noticeably in this channel, is also determined by fitting to a template obtained by reversing cuts on the lepton isolation.

Higgs boson candidates are searched for by looking for an excess in the invariant mass distribution of the two $b$-tagged jets $\left(m_{b \bar{b}}\right)$, which are shown in the Figure 2.
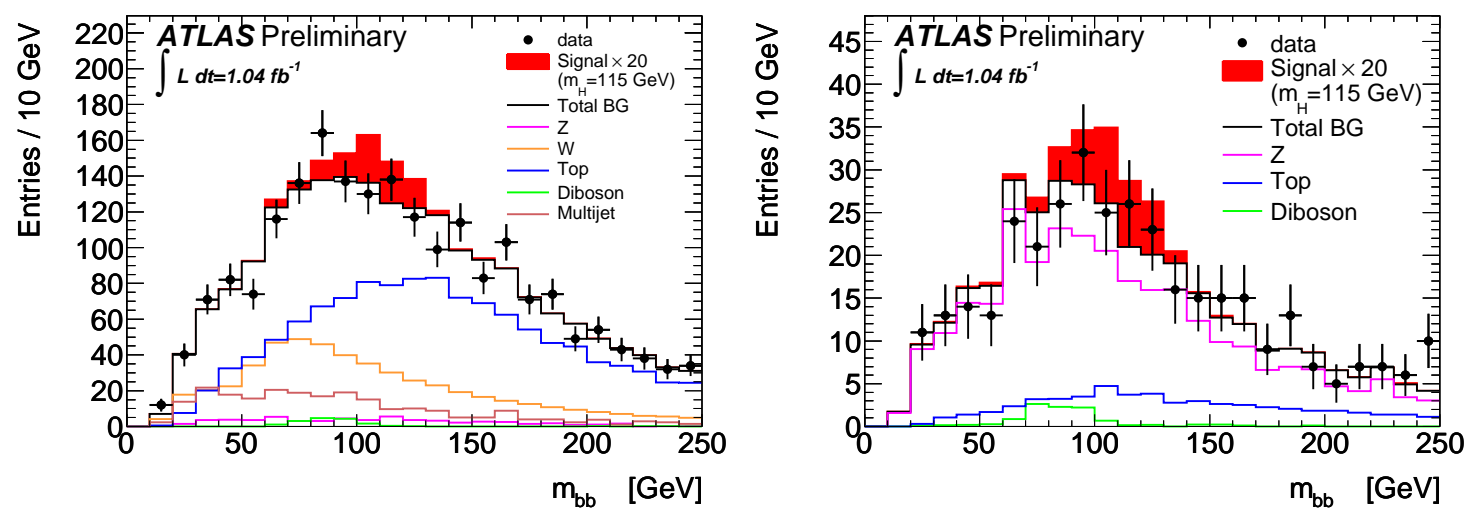

Figure 2: Left: The invariant mass, $m_{b \bar{b}}$, for $W H \rightarrow l v b \bar{b}$ and $Z H \rightarrow l l b \bar{b}$ (right) for $M_{H}=115 \mathrm{GeV}$. The signal distribution is enhanced by a factor 20 for visibility [5].

\section{2 $H \rightarrow \tau^{+} \tau^{-}$}

The search for the Higgs boson into two tau-leptons have been done in two different decay channels in the mass range between $100 \mathrm{GeV}$ and $150 \mathrm{GeV}$. The $H \rightarrow \tau \tau \rightarrow l \tau_{\text {had }} 3 v$ (the symbol $\tau_{\text {had }}$ represents that the tau lepton decays hadronically) performed with an integrated luminosity of $1.06 \mathrm{fb}^{-1}$. The event selection in this analysis requires one lepton (l) (with transverse momentum in excess of $20 \mathrm{GeV}$ for muons and $25 \mathrm{GeV}$ for electrons) from a fully leptonic tau decay, an oppositely charged tau candidate $\tau_{\text {had }}$ with transverse momentum larger than $20 \mathrm{GeV}$, selected using a calorimeter jet associated to one or more three tracks and a missing transverse energy larger than $20 \mathrm{GeV}$ due to the three undetected neutrinos. Events with an additional lepton are removed to suppress the $Z / \gamma^{*} \rightarrow l l$ and $t \bar{t}$ background processes. Finally, the $W \rightarrow l v$ background process is suppressed requiring than the transverse mass of the lepton and missing energy system to be smaller than $30 \mathrm{GeV}$. This analysis uses a new reconstruction technique to estimate the invariant mass of the pair of tau leptons, which does not assume a strict collinearity between the visible and invisible decay products of the tau leptons [25]. The main background in this analysis is the 
$Z / \gamma^{*} \rightarrow \tau^{+} \tau^{-}$process. Its invariant mass shape is estimated using an embedding technique where muons from $Z$ decay events are substituted by simulated tau decays. The other backgrounds are in part estimated using same sign events and in part using the Monte Carlo for the different between the number of opposite sign and same sign events.

On the other hand, the $H \rightarrow l^{+} l^{-}+4 v$ final state search is characterised by a back-to-back configuration of the two tau leptons in the Higgs boson rest frame. Requiring the presence of an extra jet of high transverse momentum in the event, the Higgs boson acquires a boost in the transverse plane. So, the event selection requires the presence of exactly two isolated leptons of opposite charge with an invariant mass of the dilepton system above $20 \mathrm{GeV}$. The missing transverse energy have to be above certain threshold, which dependent of the lepton pair flavor. Events are required to have a least one jet with $p_{T}$ above of $40 \mathrm{GeV}$ (see Figure 3 (left)). Additional cuts on $m_{l l}, \Delta \phi_{l l}$, $m_{\tau \tau j}$ and $m_{\tau \tau}$ are applied in order to reduce the background contributions (see Ref. [6]). The main background is the irreducible $Z / \gamma^{*} \rightarrow \tau^{+} \tau^{-}$. It uses data driven estimations, obtained with the $\tau$-embedding method from data control samples which have been validated with full simulation studies. The contribution of the $t \bar{t}$, single- $t, Z \rightarrow l^{+} l^{-}(l=e, \mu)$ and electroweak di-boson production backgrounds are estimated from simulation. The $t \bar{t}$ and $Z \rightarrow l^{+} l^{-}$backgrounds have been confirmed from data by selecting control regions enriched in these background processes. The final discriminant is the invariant mass of $\tau$-lepton system, it is computed in the collinear approximation [26], it is assumed that the neutrinos are produced from the $\tau$ decay along the direction of the visible leptons. The $m_{\tau \tau}$ distribution for this search channel is shown in Figure 3 (right).
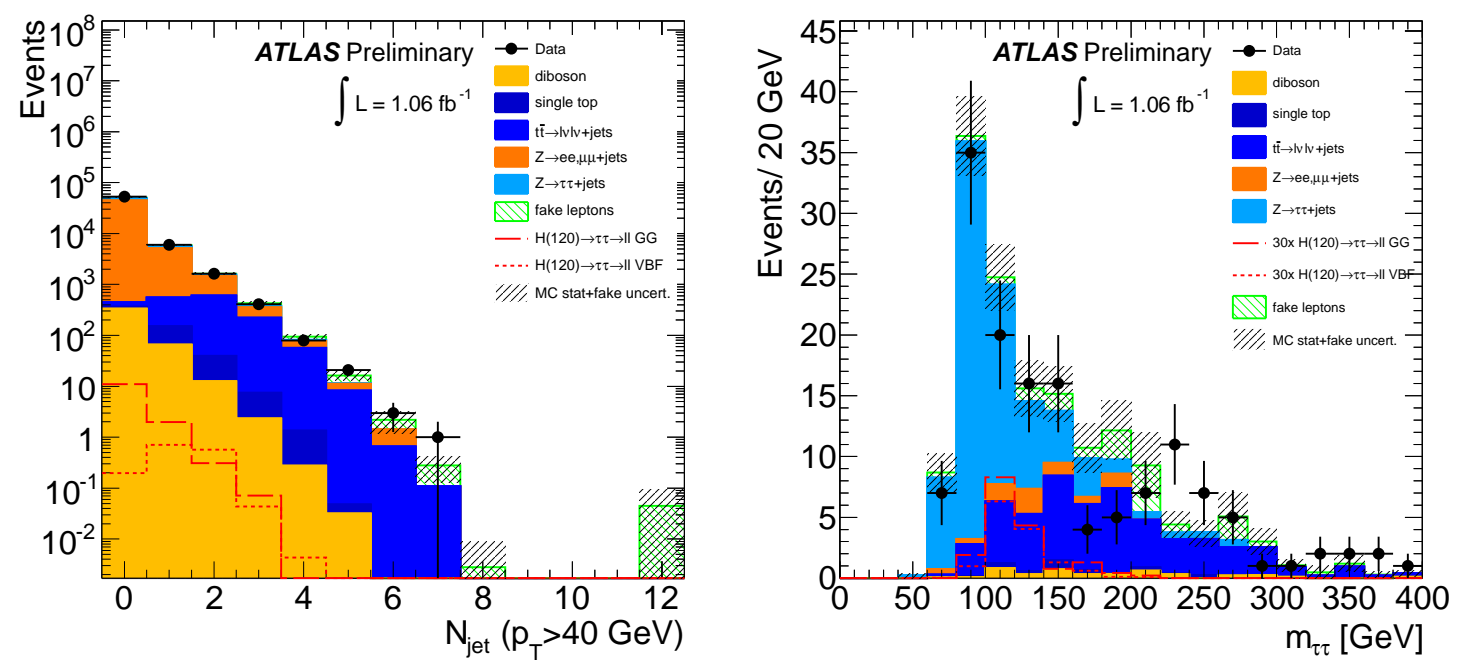

Figure 3: Left: comparison of the jet multiplicity distribution with $p_{T}^{\text {jet }}>40 \mathrm{GeV}$ between data and MC after cuts on the dilepton mass and opposite charge selection. Right: $m_{\tau \tau}$ mass after the analysis cuts for $e e, \mu \mu$ and $e \mu$ channels. The backgrounds with fake leptons and the $Z \rightarrow \tau^{+} \tau^{-}$contribution are estimated from data. All other contributions are estimated using simulated event samples [6].

\section{$4.3 H \rightarrow \gamma \gamma$}

This analysis is carried out for the Higgs boson mass hypothesis between $100 \mathrm{GeV}$ and 150 
$\mathrm{GeV}$ and uses an integrated luminosity of $1.08 \mathrm{fb}^{-1}$ [7]. This search channel selects at least two identified photons, including converted photons, isolated from any activity in the calorimeter. The leading and the sub-leading photons are required to have transverse momenta above $40 \mathrm{GeV}$ and $25 \mathrm{GeV}$, respectively. The directions of the photons are measured using the position determined in the first and second sampling of the EM calorimeter. In addition, given the non-negligible level of pileup in the 2011 data, the determination of the primary vertex position is based only on the photon direction candidates. The diphoton channel has been improved with respect to the published analysis [24], by separating the selected events into five independent categories based on the direction in which each photon was emitted (and hence with detector resolution and different signal-to-background ratio) and its photon to electron-pair conversion status (i.e. whether or not it is reconstructed as a conversion ). These categories are treated as independent channels with fully correlated systematic uncertainties.

The main background, made from genuine prompt di-photon events and reducible background are $\gamma j$ and $j j$, with one or two jets misidentified as a photon, has been measured by the extrapolation of background-rich control regions into the signal region, using the calorimeter isolation and the quality of the photon identification. The contribution of the Drell-Yan background $Z / \gamma^{*} \rightarrow e^{+} e^{-}$ with electrons misidentified as photons, is measured in the region of the $Z$ boson mass peak and extrapolated into the signal region beyond $100 \mathrm{GeV}$. Figure 4 (left) shows the extracted components of the diphoton, photon-jet, dijet and Drell-Yan processes. The purity of the sample (fraction of diphoton events) is about $75 \%$. The measurement of purity has also been made separately in each category, and ranges from $69 \%$ to $83 \%$. Figure 4 (right) shows the reconstructed diphoton mass spectrum. No excess is visible.
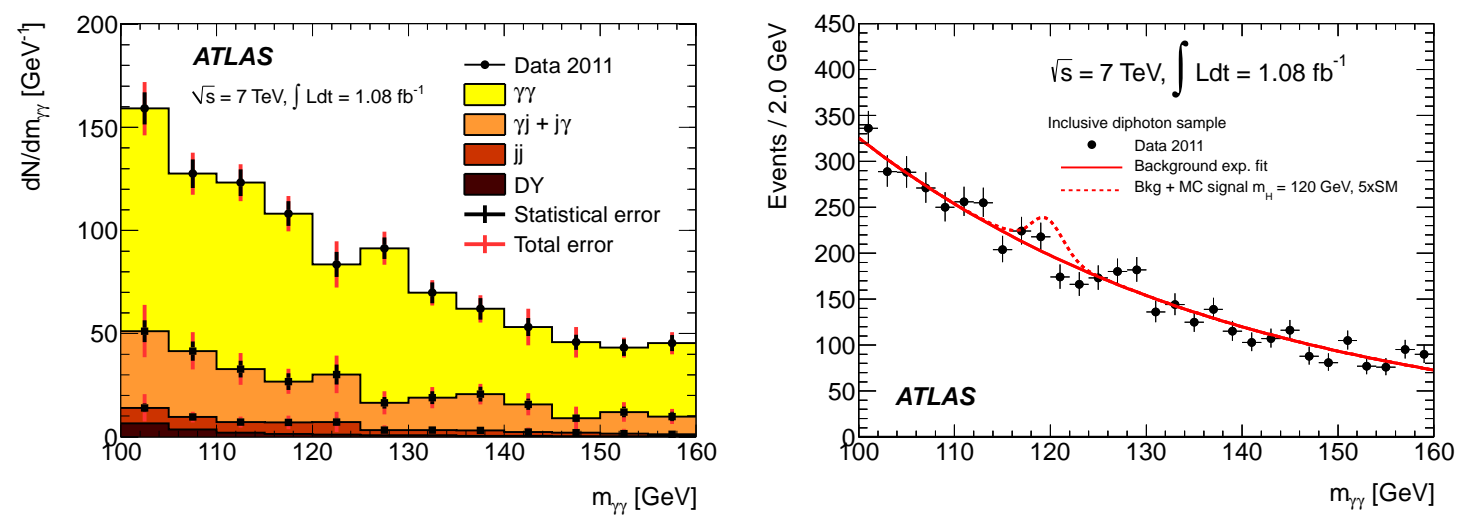

Figure 4: Left: each component of the diphoton candidate invariant mass distribution, as obtained from a data-driven method. The components are stacked on top of each other. Right: distribution of the reconstructed diphoton invariant mass for all categories together. The exponential fit to the full sample of the background-only hypothesis, as well as the expected signal for a Higgs boson mass of $120 \mathrm{GeV}$ with five times the SM predicted yield, are also shown for illustration [7].

\section{4 $H \rightarrow W W^{(*)} \rightarrow l v l v$}

This search is performed as a counting analysis for a Higgs boson mass hypotheses between 
$110 \mathrm{GeV}$ and $300 \mathrm{GeV}^{3}$, and using an integrated luminosity of $1.70 \mathrm{fb}^{-1}$ [8]. The selection requests exactly two isolated oppositely charged leptons, missing transverse energy have to be above $30 \mathrm{GeV}$. To take advantage of the angular correlations between the leptons from the spin0 nature of the Higgs, the azimuthal angle $\Delta \phi_{l l}$ is required to be below a certain threshold. The analysis is separated in 0-jet and 1-jet channels (the two or more jets channel is not included in this combination). To suppress the background form top-quark production in the 1-jet channel, events are rejected if the jet is tagged as a $b$-quark jet.

The main background contributions stem from $W W$ and top-quark production. The first is measured in a control region defined essentially by a cut on the dilepton invariant mass $m_{l l}$, requiring it to exceed $80 \mathrm{GeV}$. The second background is estimated from control regions where $b$-jet veto is reversed and the kinematic selection is relaxed. For the top control region in the 0 -jet channel the jet veto is also removed. The relative fractions of the background contributions expected in the control and signal regions are taken from Monte Carlo simulation. The other backgrounds such as $W+$ jets, $Z / \gamma^{*}+$ jets, albeit significantly smaller, are also measured in control samples of data. The small $W Z$ and $Z Z$ backgrounds are taken from Monte Carlo simulation. The transverse mass distributions of the candidates in the 0-jet and 1-jet channels are displayed in Figure 5.
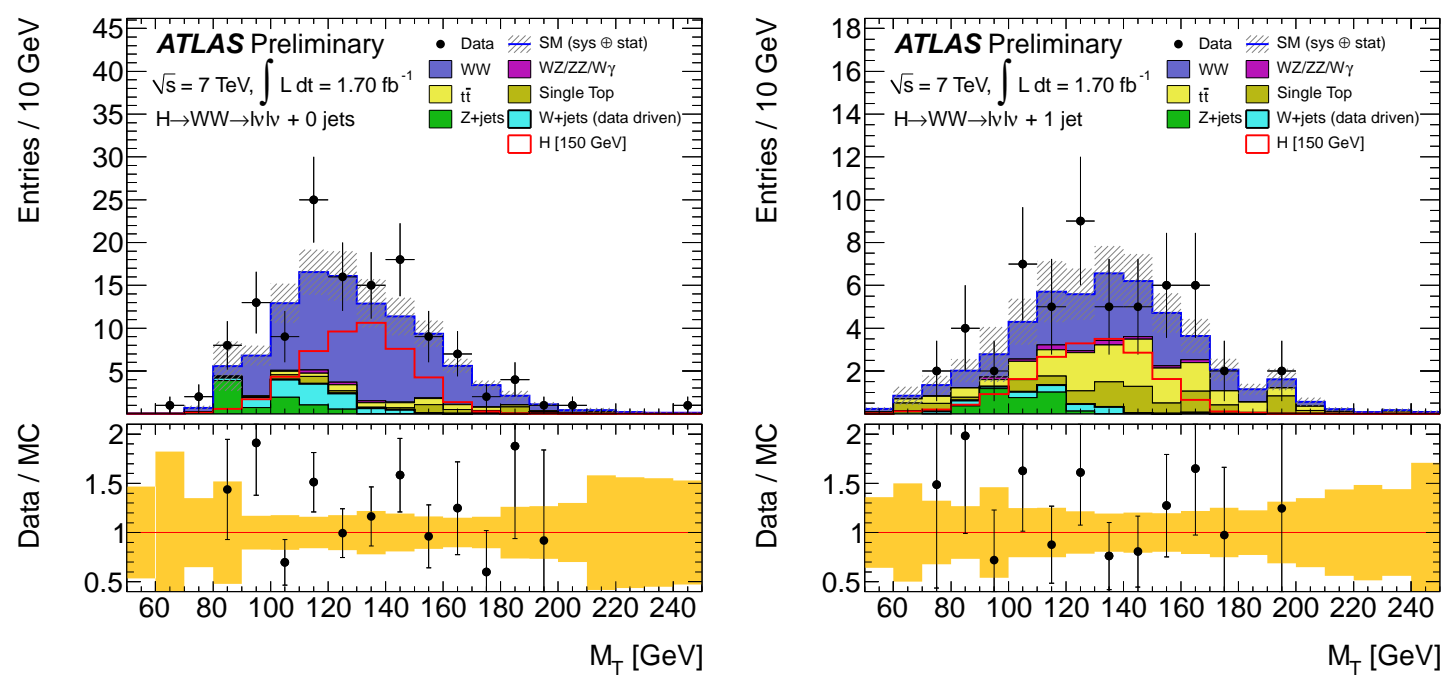

Figure 5: The transverse mass $m_{T}$ distribution in the $\mathrm{H}+0$ (left) and $\mathrm{H}+1$ (right) jet analysis after all the cuts except the cut on the $m_{T}$ itself. The expected signal is shown for $M_{H}=150 \mathrm{GeV}$. The lower part of the plot shows the ratio between the data and the background expectation from $\mathrm{MC}$, with the yellow band indicating the total systematic uncertainty in the normalization (but not the shape) of the various components. The final bin includes the overflow [8].

\section{$4.5 H \rightarrow W W \rightarrow l v q q$}

The search in this channel covers the Higgs boson mass hypothesis in the $240 \mathrm{GeV}$ to $600 \mathrm{GeV}$ range and was performed using data corresponding to an integrated luminosity of $1.04 \mathrm{fb}^{-1}$ [9]. The

\footnotetext{
${ }^{3}$ With an optimised selection in the high mass region, above $220 \mathrm{GeV}$.
} 
event selection requires the presence of exactly one lepton in the final state. Missing transverse energy is required to be above $30 \mathrm{GeV}$. This channel is separated into 0 -jet and 1-jet categories, i.e. zero and one jet in addition to the two jets from the $W$ decay. A $b$-jet veto is applied in the 1 -jet channel. The background originates overwhelmingly from the $W+$ jets process. Using the $W$ mass constraint on the leptonic $W$ decay allows good reconstruction of the invariant mass of the $W W$ system. The resolution is sufficient to directly fit the background normalization from the data with a double exponential model. The final discriminant variable is the four-object invariant mass $m_{l v q q}$ with we constraint $m_{l v}$ to be consistent with the mass of the $W$ boson. The $m_{l v q q}$ distribution of the selected candidates is illustrated in Figure 6 (left), where the expected signal for $M_{H}=400 \mathrm{GeV}$ is also shown. In Figure 6 (right) is shown the difference between the data and fitted non-resonant background.
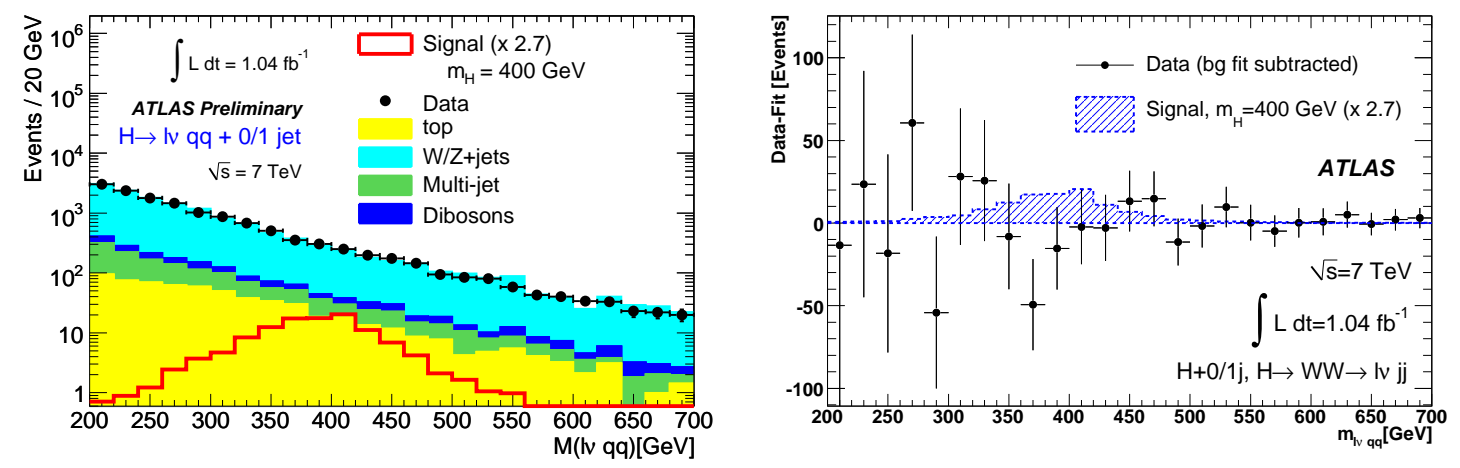

Figure 6: Left: the reconstructed invariant mass $m_{l v q q}$ in the data summed over lepton flavor and jet multiplicity. The expected backgrounds are also shown. Right: the difference between data and the fitted non-resonant background. The expected contribution from Higgs boson decays $M_{H}=400 \mathrm{GeV}$ in the SM is also shown, multiplied by a factor of 2.7 [9].

\section{6 $H \rightarrow Z Z^{(*)} \rightarrow 4 l$}

The so-called "gold-plated" channel in the Higgs boson search because its benefits from a fully reconstructed final state, which leads to a narrow four-lepton invariant mass peak on the top of a continuous background, but has small rates. The selection requires two pairs of oppositely charged and isolated same-flavour leptons, with one di-lepton mass compatible with the $\mathrm{Z}$ boson mass $\left(m_{12}\right)$ and the second di-lepton mass $\left(m_{34}\right)$ exceeding a certain threshold depending on the Higgs boson mass. The reducible $Z+$ jets and top-quark backgrounds, which have a larger impact in the low invariant mass range, are estimated from data control regions, while the main irreducible $Z Z^{(*)}$ background was estimated from Monte Carlo. Results are given for Higgs boson mass hypotheses in the $110 \mathrm{GeV}$ to $600 \mathrm{GeV}$ range and are using data corresponding to integrated luminosities of 2.0-2.3 $\mathrm{fb}^{-1}$ [10]. Twenty-seven candidates were observed. The $m_{12}, m_{34}$, and $m_{4 l}$ mass spectra are shown in Figure 7. The $m_{4 l}$ distribution for the total background and several signal hypotheses is compared to the data in left plate of Figure 7. 

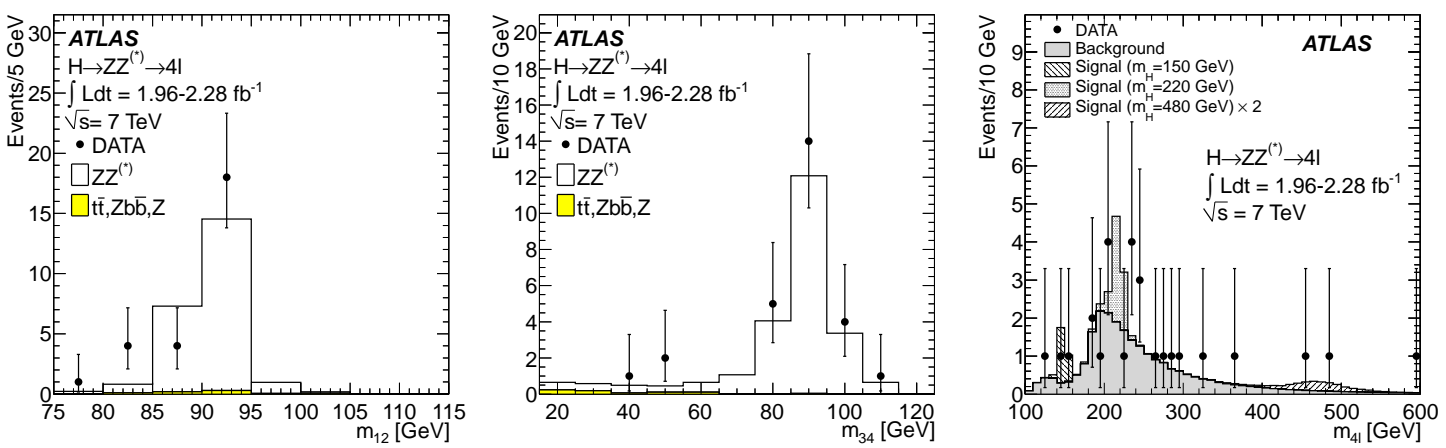

Figure 7: Invariant mass distributions $m_{12}, m_{34}$, and $m_{4 l}$ for the selected candidates. The data (dots) are compared to the background expectations from the dominant $Z Z^{(*)}$ process and the sum of $t \bar{t}, Z b \bar{b}$ and $Z+$ jets processes. Error bars represent $68.3 \%$ central confidence intervals. Left: the $m_{4 l}$ distribution, the signal expectation for three $M_{H}$ hypotheses is also shown as well as the total background [10].

\section{7 $H \rightarrow Z Z \rightarrow l l v v$}

The analysis in this channel is carried out in the $200 \mathrm{GeV}$ to $600 \mathrm{GeV}$ mass range of the Higgs boson hypothesis using data corresponding to an integrated luminosity of $1.04 \mathrm{fb}^{-1}$ [12]. There are two search regions, with $280 \mathrm{GeV}$ and upwards considered to be high mass. The event selection requires two high $p_{T}$ isolated leptons, a minimum amount of missing transverse energy in the event as well as the azimuthal di-lepton angle $\Delta \phi_{l l}$ to be below a certain threshold. The $b$-tagged jet as well as the $3^{\text {rd }}$ lepton are vetoed to reduce the top background. In addition, the selection is protected from a potential overlap with the $H \rightarrow W W^{(*)} \rightarrow l v l v$ channel by selecting events for which the invariant mass of the dilepton system is consistent with the $Z$ mass. The main irreducible background in this analysis is the $Z Z$ production process, which is fully estimated from the Monte Carlo simulation, as are $W W$ and $W Z$ backgrounds. The $Z+$ jets and top-quark production backgrounds are also taken from the Monte Carlo but with further verifications in data control regions. The $W+$ jets normalization is obtained from a control region.

\section{$4.8 H \rightarrow Z Z \rightarrow l l q q$}

This analysis is performed in the mass range of $200 \mathrm{GeV}$ to $600 \mathrm{GeV}$ and it is separated into a low and a high mass region where the search criteria are independently optimized, the separation occurs at $280 \mathrm{GeV}$. Given the sizable branching fraction of the $Z$ decaying into a pair of $b$-quarks ( $\sim 15 \%$, which is much larger than the fraction of $b$-quark jets in the background) the analysis is separated into two categories: the tagged analysis where the two jets are $b$-tagged and the untagged analysis where at most one of the jets is $b$-tagged. The dijet system is scaled to set its invariant mass to that of the $Z$ boson in order to improve the mass resolution of the $l l q q$ system. This channel uses data corresponding to an integrated luminosity of $1.04 \mathrm{fb}^{-1}$ [11]. The dominant background in this analysis is the $Z+$ jets production process. Its normalization is measured in the sidebands of the dijet mass distribution. The other backgrounds are estimated with the Monte Carlo simulation and verified with data control samples for the top-quark background. The dilepton-dijet invariant mass is used as the discriminant and its shape is estimated using Monte Carlo simulation both for 
the signal and the backgrounds. The acceptance of this channel overlaps significantly with that of the $H \rightarrow b \bar{b}$ analysis, however as these two channels span different ranges in Higgs boson mass hypotheses they are disjoined.

\section{Combined Results}

The combination procedure follows precisely that described in Refs. [24, 27, 28]. It is based on the profile likelihood test statistic [13]. The 95\% CL cross section limits in units of the SM expectation set by individual channels are shown in Figure 8. The combination of all channels is shown in Figure 9 in terms of the observed and the expected upper limit at a 95\% CL on the excluded Higgs boson production cross section, normalised to the SM value. The limits shown are made using the asymptotic approximation [13], which have been verified using Monte Carlo experiments and a Bayesian calculation, which agrees with these results to within a few percent [27]. The expected exclusion covers the SM Higgs boson mass range from $131 \mathrm{GeV}$ to $447 \mathrm{GeV}$. The observed $95 \% \mathrm{CL}$ exclusion regions are from $146 \mathrm{GeV}$ to $232 \mathrm{GeV}, 256 \mathrm{GeV}$ to $282 \mathrm{GeV}$ and 296 $\mathrm{GeV}$ to $466 \mathrm{GeV}$. A deficit of events observed in the excluded mass range and in particular between 300 to $400 \mathrm{GeV}$ as reported in Ref. [27] is unchanged and still due to the concordance of various small deficits in several high mass channels. The observed limits is in good agreement with the background only expectation over most of the available range in Higgs boson mass hypotheses, except in the low mass range (from $\sim 130 \mathrm{GeV}$ up to $\sim 170 \mathrm{GeV}$ ), range where a slight excess is still observed.

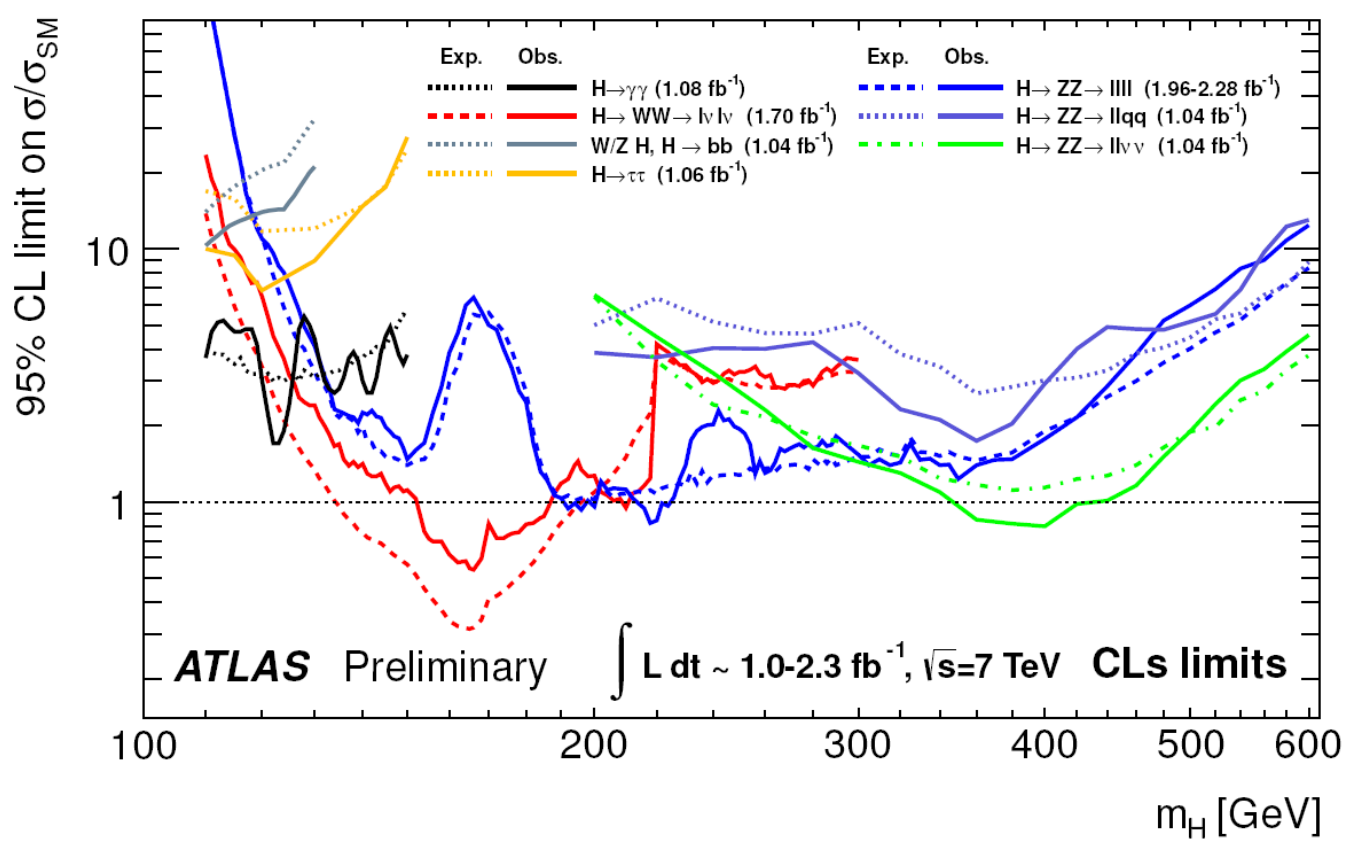

Figure 8: The expected (dashed) and observed (solid) cross section limits for the individual search channels, normalised to the Standard Model Higgs boson cross section, as functions of the Higgs boson mass. These results use the profile likelihood technique with 95\% CL limits using the CLs construction [34]. 


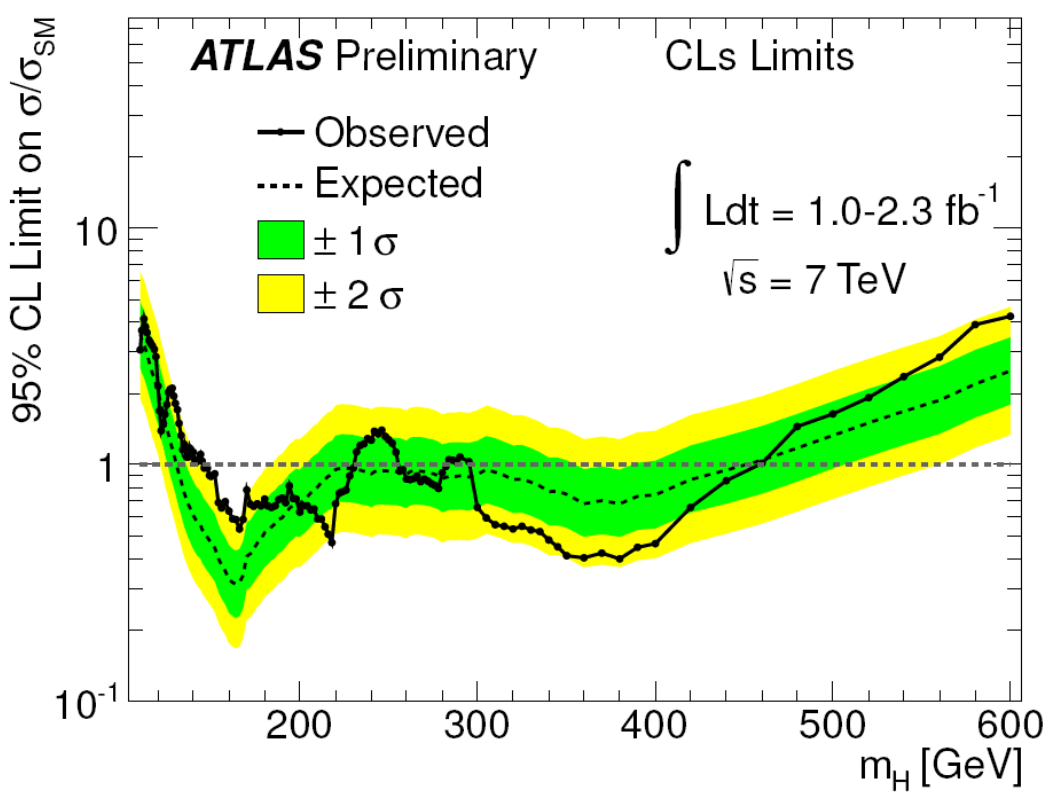

Figure 9: The combined upper limit on the Standard Model Higgs boson production cross section divided by the Standard Model expectation as a function of $\mathrm{mH}$ is indicated by the solid line. This is a 95\% CL limit using the CLs method in the full mass range of this analysis. The dotted line shows the median expected limit in the absence of a signal and the green and yellow bands indicate the corresponding $68 \%$ and $95 \%$ expected regions [34].

\section{Search for the neutral MSSM Higgs bosons}

In this section, a search for neutral Higgs bosons decaying to pairs of $\tau$-leptons is presented. Four different di- $\tau$ decay final states, $\tau \tau \rightarrow e \mu 4 v, e \tau_{\text {had }} 3 v, \mu \tau_{\text {had }} 3 v$ and $\tau_{\text {had }} \tau_{\text {had }} 2 v$, are considered. This analysis was performed using a data sample corresponding to an integrated luminosity of $1.06 \mathrm{fb}^{-1}$ [29].

The event selection in the $e \mu 4 v$ signal events corresponds to one isolated electron, one isolated muon and missing transverse momentum due to the undetected neutrinos from the two $\tau$ decays, where either one of the leptons can trigger the event. If the event was triggered by the electron trigger a reconstructed electron with $p_{T}^{e}>22 \mathrm{GeV}$ and a muon with $p_{T}^{\mu}>10 \mathrm{GeV}$ with opposite electric charge is required. Otherwise, if the event was $\mu$ triggered by the muon trigger only it is required that there is a reconstructed muon with $p_{T}^{\mu}>20 \mathrm{GeV}$ and a reconstructed electron with $p_{T}^{e}>15 \mathrm{GeV}$ with opposite electric charge. The $t \bar{t}$, single-top and di-boson backgrounds are suppressed asking the following two additional requirements: the scalar sum of the transverse momentum of the electron, the transverse momentum of the muon and the missing transverse momentum must be smaller than $120 \mathrm{GeV}$, and the azimuthal opening angle between the electron and the muon must be larger than $2.0 \mathrm{rad}$.

The signatures of $A / H / h \rightarrow e / \mu \tau_{\text {had }} 3 v$ signal events, ask that one $\tau$ lepton decays leptonically (high- $p_{T}$ isolated $e$ or $\mu$ ) and the other hadronically, with missing transverse momentum. The $p_{T}$ of the lepton has to be bigger than a certain threshold, this cut being dependent of the lepton 
flavour. One oppositely-charged $\tau_{\text {had }}$ candidate with $p_{T, \tau_{\text {had }}}>20 \mathrm{GeV}$ is also required in the event. Events with more than one electron or muon reconstructed, using less strict requirements on the leptons, are rejected to suppress events from $Z / \gamma^{*} \rightarrow l^{+} l^{-}(l=e, \mu)$ decays and from $t \bar{t}$ or singletop production. Events with jets from QCD processes as well $Z / \gamma^{*} \rightarrow l^{+} l^{-}(l=e, \mu)$ decays are rejected by requiring $E_{T}^{\text {miss }}>20 \mathrm{GeV}$. Events with real leptons from $W \rightarrow l v$ decays are suppressed by requiring the transverse mass of the $E_{T}^{\text {miss }}$ and the lepton to be smaller than $30 \mathrm{GeV}$.

The $A / H / h \rightarrow \tau^{+} \tau^{-} \rightarrow \tau_{\text {had }} \tau_{\text {had }} \nu v$ signature is characterized by two identified hadronic tau decays and missing transverse momentum from the undetected neutrinos. Events with exactly two oppositely charged $\tau_{\text {had }}$ candidates that match a dedicated $\tau_{\text {had }}$ trigger are selected. The leading and sub-leading $\tau_{\text {had }}$ candidates must have $p_{T}$ bigger than $45 \mathrm{GeV}$ and $30 \mathrm{GeV}$, respectively. To suppress backgrounds from $Z, W$ boson production and QCD multijet production the missing transverse momentum is required to be bigger than $25 \mathrm{GeV}$. Finally, events are rejected if they contain an electron candidate with $p_{T}^{e}>15 \mathrm{GeV}$ or a muon candidate with $p_{T}^{\mu}>10 \mathrm{GeV}$, isolation is not required.
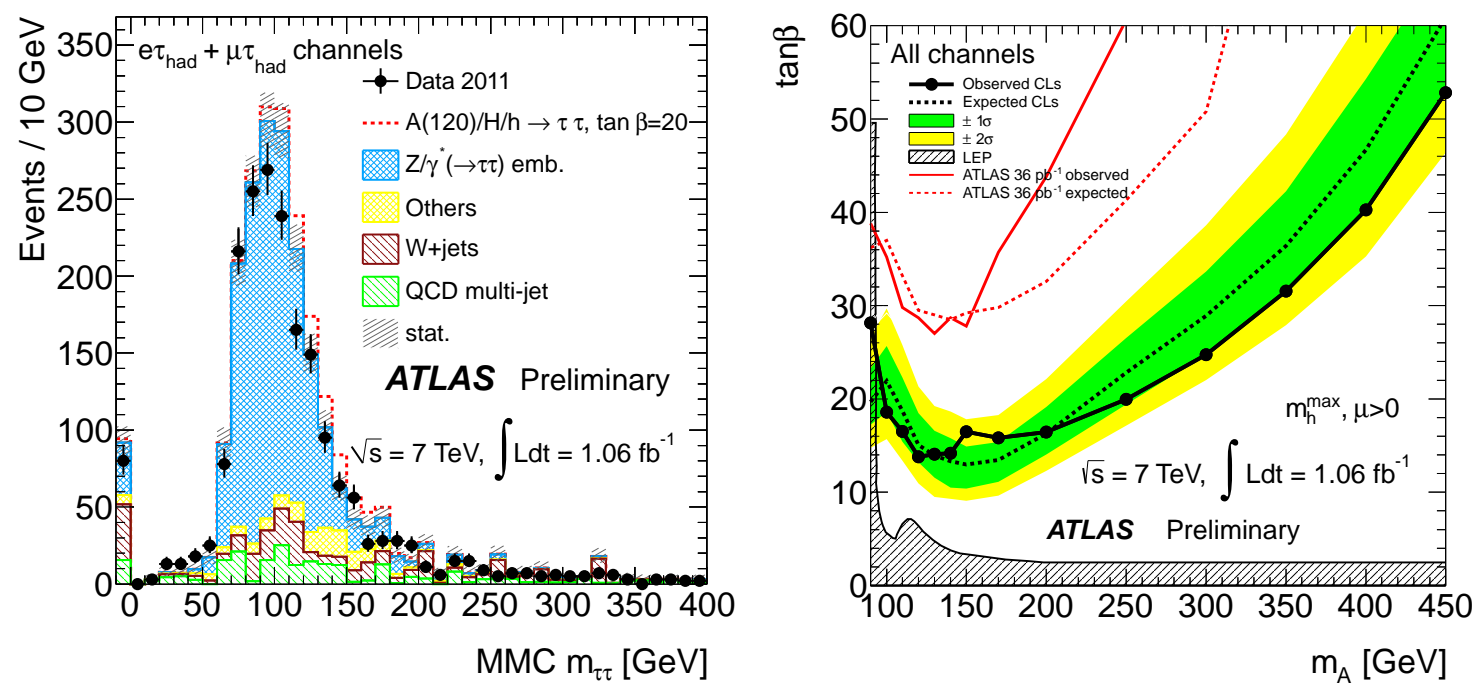

Figure 10: Left: shows the di-tau mass distribution computed with the MMC tehcnique [25] for $e \tau_{\text {had }}$ and $e \tau_{\text {had }}$ final state channels. Right: expected and observed exclusion limits based on CLs in the $m_{A}-\tan$ beta plane of the MSSM derived from the combination of the analyses for the $e, \mu, l, \tau_{\text {had }}$ and $\tau_{\text {had }}, \tau_{\text {had }}$ final states. The exclusion limits from a previous result and from LEP are also shown [29].

After the selection of signal candidates, different $\tau^{+} \tau^{-}$mass reconstruction methods are studied, which are well documented in Ref. [29]. For example, in the Figure 10 (left) is shown the transverse mass of the di-tau system using the technique described in Ref. [25] for the $e / \mu \tau_{\text {had }}$ final states. The background estimation uses similar techniques to those described in Subsection 4.2

No significant excess of events is found. Exclusion limits at the $95 \%$ confidence level are set on the production cross section times branching ratio, $\sigma \times B R\left(\phi \rightarrow \tau^{+} \tau^{-}\right)$, of a generic Higgs boson $\phi$ as a function of its mass, $m_{\phi}$, and for MSSM Higgs boson $A / H / h$ production as a function of the parameters $m_{A}$ and $\tan \beta$ (see Figure 10 (right)). The exclusion limits are derived with the profile likelihood method [13] based on the CLs parameter. 


\section{Conclusions}

The outstanding performance of the LHC in 2011 allows for great sensitivity in the combined ATLAS searches for the SM and beyond SM Higgs boson. With the amount of analysed data, no significant evidence of a signal has yet been observed, although an excess corresponding to a $2.8 \sigma$ fluctuation (without taking into account the look Elsewhere effect) of the background occurs in the SM Higgs boson mass range between $130 \mathrm{GeV}$ and $150 \mathrm{GeV}$.

The SM Higgs boson is excluded at 95\% CL in a hitherto unconstrained range of Higgs boson mass hypotheses in the range from 146 to $232 \mathrm{GeV}, 256$ to $282 \mathrm{GeV}$ and 296 to $466 \mathrm{GeV}$. This is in good agreement with the sensitivity of this search. The excluded region starts to cover a large fraction of the allowed mass range of the SM Higgs boson.

In addition, exclusion limits on both the cross section for the production of a generic Higgs boson $\phi$ as a function of its mass and on MSSM Higgs boson production $A / H / h$ as a function of $m_{A}$ and $\tan \beta$, are derived. These results exclude regions of parameters space beyond the existing limits from previous experiments at LEP [30] and the Tevatron [31, 32] and are similar to those recently obtained by the CMS Collaboration [33].

\section{References}

[1] LEP Working Group for Higgs boson searches, R. Barate et al., Search for the Standard Model Higgs boson at LEP, Phys. Lett. B565 (2003) 61-75.

[2] The TEVNPH Working Group of the CDF and DØ Collaboration, Combined CDF and $D \varnothing$ upper limits on Standard Model Higgs-boson production with up to $6.7 \mathrm{fb}^{-1}$ of data, arXiv:1007.4587 [hep-ex].

[3] The TEVNPH Working Group of the CDF and DØ Collaboration, Combined CDF and $D \emptyset$ upper limits on Standard Model Higgs-boson production with up to $8.2 \mathrm{fb}^{-1}$ of data, arXiv:1103.3233 [hep-ex].

[4] The Gfitter Group, M. Baak et al., Updated Status of the Global Electroweak Fit and Constrains on New Physics, CERN-PH-EP-2011-033 (CERN, Geneva, 2011), arXiv:1107.0975 [hep-ex].

[5] The ATLAS Collaboration, Search for the Standard Model Higgs boson produced in association with a vector boson and decaying to a b-quark pair with the ATLAS detector at the LHC, ATLAS-CONF-2011-103, (CERN, Geneva, July 2011).

[6] The ATLAS Collaboration, Search for the Standard Model Higgs boson in the Decay Mode $H \rightarrow \tau^{+} \tau^{-} \rightarrow l l+4$ Neutrinos in Association with Jets in Proton-Proton Collisions at $\sqrt{s}=7$ $\mathrm{TeV}$ with the ATLAS Detector, ATLAS-CONF-2011-133, (CERN, Geneva, August 2011).

[7] The ATLAS Collaboration, Search for the Standard Model Higgs boson in the two photon decay channel with the ATLAS detector at the LHC, CERN-PH-EP-2011-129 (CERN, Geneva, 2011), arXiv:1108.5895 [hep-ph]. 
[8] The ATLAS Collaboration, Search for the Standard Model Higgs boson in the $H \rightarrow W W^{(*)} \rightarrow$ lv lv decay mode using $1.7 \mathrm{fb}^{-1}$ of data collected with the ATLAS detector at $\sqrt{s}=7 \mathrm{TeV}$, ATLAS-CONF-2011-134 (CERN, Geneva, 2011).

[9] The ATLAS Collaboration, Search for the Higgs boson in the $H \rightarrow W W \rightarrow l v j j$ decay channel in pp collisions at $\sqrt{s}=7 \mathrm{TeV}$ with the ATLAS detector CERN-PH-EP-2011-130 (CERN, Geneva, 2011), arXiv:1109.3615 [hep-ph].

[10] The ATLAS Collaboration, Search for the Standard Model Higgs boson in the decay channel $H \rightarrow 4 l$ with the ATLAS detector, CERN-PH-EP-2011-144 (CERN, Geneva, 2011), arXiv:1109.5945 [hep-ph].

[11] The ATLAS Collaboration, Search for a heavy Standard Model Higgs boson in the channel $H \rightarrow l l q q$ using the ATLAS detector, CERN-PH-EP-2011-136 (CERN, Geneva, 2011), arXiv:1108.5064 [hep-ph].

[12] The ATLAS Collaboration, Search for a Standard Model Higgs boson in the $H \rightarrow l l v v$ decay channel with the ATLAS detector, CERN-PH-EP-2011-142 (CERN, Geneva, 2011), arXiv:1109.3357 [hep-ph].

[13] G. Cowan, K. Cranmer, E. Gross and O. Vitells, Asymptotic formulae for likelihood-based tests of new physics, Eur. Phys. J. C71 (2011) 1-19.

[14] S. Dittmaier, C. Mariotti, G. Passarino, and R. Tanaka (Eds.), LHC Higgs Cross Section Working Group, Handbook of LHC Higgs Cross Sections: 1. Inclusive Observables, CERNPH-EP-2011-002 (CERN, Geneva, 2011), arXiv:1101.0593 [hep-ph] .

[15] Charalampos Anastasiou and Kirill Melnikov, Higgs boson production at hadron colliders in NNLO QCD, Nucl. Phys., B646:220-256, 2002.

[16] Robert V. Harlander and William B. Kilgore, Next-to-next-to-leading order Higgs production at hadron colliders, Phys. Rev. Lett., 88:201801, 2002.

[17] V. Ravindran, J. Smith, and W. L. van Neerven, NNLO corrections to the total cross section for Higgs boson production in hadron hadron collisions, Nucl. Phys., B665:325-366, 2003.

[18] Paolo Bolzoni, Fabio Maltoni, Sven-Olaf Moch, and Marco Zaro, Higgs production via vector-boson fusion at NNLO in QCD, Phys. Rev. Lett., 105:011801, 2010.

[19] O. Brein, A. Djouadi, and R. Harlander, NNLO QCD corrections to the Higgsstrahlung processes at hadron colliders, Physics Letters B, 579:149-156, 2004.

[20] W. Beenakker et al, Higgs radiation off top quarks at the Tevatron and the LHC, Phys. Rev. Lett., 87:201805, 2001.

[21] W. Beenakker et al, NLO QCD corrections to $t$ anti-t $H$ production in hadron collisions, Nucl. Phys., B653:151-203, 2003. 
[22] S. Dawson, L. H. Orr, L. Reina, and D. Wackeroth, Associated top quark Higgs boson production at the LHC, Phys. Rev., D67:071503, 2003.

[23] ATLAS Collaboration, The ATLAS Experiment at the CERN Large Hadron Collider, JINST 3 (2008) S08003.

[24] ATLAS Collaboration, Limits on the production of the Standard Model Higgs Boson in pp collisions at $\sqrt{s}=7 \mathrm{TeV}$ with the ATLAS detector, arXiv:1106.2748 [hep-ex] .

[25] A. Elagin, P. Murat, A. Pranko, and A. Safonov, A new Mass Reconstruction Technique for Resonances Decaying to di-tau, arXiv:1012.4686 [hep-ex].

[26] R.K. Ellis, I. Hinchliffe, M. Soldate and J.J. Van der Bij, Higgs decay to $\tau^{+} \tau^{-}$: A possible signature of intermediate mass Higgs bosons at high energy hadron colliders, Nucl. Phys., B297:221-243, 1988.

[27] ATLAS Collaboration, Combination of the Searches for the Higgs Boson in $\sim 1 \mathrm{fb}^{-1}$ of Data Taken with the ATLAS Detector at 7 TeV Center-of-Mass Energy, ATLAS-CONF-2011-112, 2011.

[28] ATLAS Collaboration and CMS Collaboration, LHC Higgs Combination Working Group Report, ATL-PHYS-PUB-2011-818, 2011.

[29] ATLAS Collaboration, Search for neutral MSSM Higgs bosons decaying to $\tau^{+} \tau^{-}$pairs in proton-proton collisions at $\sqrt{s}=7 \mathrm{TeV}$ with the ATLAS detector, ATLAS-CONF-2011-132, 2011.

[30] ALEPH Collaboration, DELPHI Collaboration, L3 Collaboration, OPAL Collaboration and LEP Working Group for Higgs Boson Searches, Eur. Phys. J. C47 (2006) 547-587.

[31] The CDF and DØ Collaborations and Tevatron New Physics Higgs Working Group (TEVNPHWG), arXiv:1003.3363 [hep-ex].

[32] The DØ Collaboration, V.M. Abazov et al., arXiv:1106.4885 [hep-ex] .

[33] The CMS Collaboration, S. Chatrchyan et al., CMS- PAS-HIG-11-009.

[34] ATLAS Collaboration, Update of the Combination of Higgs Boson Searches in 1.0 to 2.3 $\mathrm{fb}^{-1}$ of pp Collisions Data Taken at $\sqrt{s}=7 \mathrm{TeV}$ with the ATLAS Experiment at the LHC, ATLAS-CONF-2011-135, 2011. 